\title{
An Exploration of the Active Ingredients of Cortex phellodendri in the Treatment of Hemorrhoids and Its Mechanism Based on Network Pharmacology
}

\author{
Xianchang Yu, Xiongdong Zhong* \\ Email address: \\ 454009703@qq.com (Xianchang Yu), zhongxiongdong@sina.com (Xiongdong Zhong) \\ ${ }^{*}$ Corresponding author
}

Department of Cardiothoracic Surgery, Zhuhai People's Hospital (Zhuhai Hospital Affiliated with Jinan University), Zhuhai, P. R. China

To cite this article:

Xianchang Yu, Xiongdong Zhong. An Exploration of the Active Ingredients of Cortex phellodendri in the Treatment of Hemorrhoids and Its Mechanism Based on Network Pharmacology. Journal of Surgery. Vol. 8, No. 6, 2020, pp. 209-216. doi: 10.11648/j.js.20200806.17

Received: November 20, 2020; Accepted: November 30, 2020; Published: December 4, 2020

\begin{abstract}
Background: Huangbo, also known as Cortex phellodendri in Latin, is an important drug which has the pharmacological action of clearing heat, drying dampness, purging fire and detoxifying. In recent years, it has been reported that Huangbo also has the activity of treating hemorrhoids. Objective: The aim of this study was to explore the Active Ingredients of Cortex phellodendri in the Treatment of hemorrhoids and Its Mechanism. Study Design: We analyzed Active components and target genes of Cortex phellodendri in the Traditional Chinese Medicine System Pharmacology (TCMSP) database and analysis platform. We then searched the GeneCards database for target genes related to hemorrhoids and the intersection of these genes with the active components of Cortex phellodendri. Target genes related to hemorrhoids were taken as common potential target genes of Cortex phellodendri, which could act on hemorrhoids. Using the R programming language, we drew a Venn map of these common potential target genes. The "component-target gene-disease" network of Cortex phellodendri in the treatment of hemorrhoids was established using Cytoscape software version 3.7.1; the protein-protein interaction (PPI) network was constructed in the Search Tool for the Retrieval of Interacting Genes/Proteins (STRING) database. With the help of R and Perl languages, we performed gene ontology (GO) function and Kyoto Encyclopedia of Genes and Genomes (KEGG) pathway enrichment analyses of potential target genes of Cortex phellodendri in the treatment of hemorrhoids. Results: We extracted a total of 10 active components from Cortex phellodendri, including quercetin and rutaecarpine and so on, as well as 29 potential target genes for hemorrhoids. According to the Degree ranking in Cytoscape3.7.1 software, the top 10 potential target genes were interleukin-6 (IL-6), CCL2, CXCL8, MMP9, vascular endothelial growth factor A (VEGFA), Myc, IL-10, ICAM1, MMP2, and MMP3. Pathway enrichment mainly involved signaling pathways such as advanced glycation end products and receptor for advanced glycation end products (AGE-RAGE) signaling pathway in diabetic complications, hypoxia-inducible factor 1 (HIF-1), IL-17, and Relaxin. Conclusion: Based on network pharmacology, Cortex phellodendri is expected to be mined as a candidate Traditional Chinese Medicine (TCM) for the treatment of hemorrhoids. Its mechanism for treating this disease operates via multiple components and pathways. This study provides the basic theory and the basis for further research.
\end{abstract}

Keywords: Cortex phellodendri, Hemorrhoids, Network Pharmacology, Target Gene, GO Function Enrichment Analysis, KEGG Pathway Enrichment Analysis

\section{Introduction}

Hemorrhoids is one of the common anorectal diseases [1]. The incidence of hemorrhoids varies from region to region $[2$, 3]. According to statistics, the incidence of hemorrhoids in the United States population is 53-68\% [4]; whereas in China, it's 55 to $75 \%$ [5]. Hemorrhoids are currently treated in a variety of ways, each with its own advantages, but postoperative recurrence, bleeding, anal edema and other symptoms are still common [6]. 
Traditional Chinese Medicine (TCM) treatment of hemorrhoids has a long history and rich theoretical basis [7]. In clinical practice, TCM can effectively improve the clinical symptoms of hemorrhoids patients and reduce the incidence of serious hemorrhoids symptoms [8]. TCM has an overall regulatory effect on the body through multiple components and targets [9]. Cortex phellodendri is one of the common Chinese medicines for hemorrhoids. The medicine of Cortex phellodendri is cold and bitter. It has the effect of clearing heat, drying and dampness, relieving fire and detoxifying [10]. The theory of TCM has proved that hemorrhoids are caused by dampness and heat [11]. Therefore, with the effect of clearing heat and dampness, Cortex phellodendri has a unique important role in the treatment of hemorrhoids [12]. However, the active ingredients and mechanism of Cortex phellodendri in the treatment of hemorrhoids are still unclear.

By integrating computer technology and bioinformatics technology, network pharmacology systematically explores the regulatory pathway of TCM on the signal pathway and selects effective targets, which can systematize, informationize and scientize the mechanism of TCM in the treatment of hemorrhoids, and has become one of the most effective methods to study the mechanism of hemorrhoids in the treatment of traditional Chinese medicine [13]. Therefore, based on network pharmacology, this study explored the effective components and mechanism of Phellodendron bark in the treatment of hemorrhoids, and preliminarily obtained a good discovery. Now the summary report is as follows.

\section{Materials and Methods}

All researches were approved by the Research Committee of Zhuhai People's Hospital (Zhuhai Hospital Affiliated with Jinan University), and carried out in accordance with the approved guidelines.

\subsection{Searching Active Components of Cortex phellodendri}

We input the keyword "Cortex phellodendri" into the Traditional Chinese Medicine System Pharmacology (TCMSP) database and analysis platform and retrieved Cortex phellodendri's active ingredients. According to recommendations in the literature, we regarded compounds with oral bioavailability (OB) $>30 \%$ and drug-likeness (DL) $>0.18$ as active components.

\subsection{Searching Potential Target Genes of Cortex phellodendri}

We searched all target genes of Cortex phellodendri and then extracted the target genes of its active components. We imported 29 potential target genes of Cortex phellodendri's active components into the UniProt database (https://www.uniprot.org/), selected "human" as the species, and obtained names of target genes and their corresponding acronyms.

\subsection{Searching for Hemorrhoids-related Genes}

The GeneCards database (http://www.genecards.org/) is a 1-stop database for human-gene annotation that covers nearly $90 \%$ of human protein-coding genes. We input the keyword "hemorrhoids" into GeneCards and then exported the retrieval results.

\subsection{Intersection of Potential Target Genes of Cortex phellodendri and Hemorrhoids-related Genes}

We considered genes that were both potential target genes of Cortex phellodendri and hemorrhoids -related genes to be the genes of interaction between Cortex phellodendri and hemorrhoids.

\subsection{Constructing a Component-target Gene-disease Network}

We deleted active components that could not act on hemorrhoids-related target genes. Then we imported the remaining active components and potential target genes for hemorrhoids treatment into Cytoscape software version 3.7.1 (US National Institute of General Medical Sciences [NIGMS], Bethesda, Maryland, US; https://cytoscape.org/) to build an active componenttarget gene-disease network.

\subsection{Constructing a Protein-protein Interaction (PPI) Network}

We input potential target genes of Cortex phellodendri in the treatment of hemorrhoids into the Search Tool for the Retrieval of Interacting Genes/Proteins (STRING) database (https://string-db.org/) and selected "Homo sapiens" as the species. We obtained the PPI network in tab-separated values (.tsv) file format; the PPI network picture was saved in Portable Network Graphics (.png) format.

\subsection{Screening Core Genes of PPI Network}

Using the $\mathrm{R}$ programming language, we calculated a genes.tsv file and selected the Top 20 target genes of degree value as the core genes.

\subsection{Gene Ontology (GO) Function and Kyoto Encyclopedia of Genes and Genomes (KEGG) pathway Enrichment Analyses}

Using $\mathrm{R}$ language, we analyzed our GO function enrichment analysis of potential target genes and our KEGG pathway enrichment analysis, including biological processes, molecular functions, and cell components. We selected the path whose kinase insert domain receptor (KDR) value (i.e., $Q$-value) was $<0.05$, and then we ranked the paths with the 20 highest KDR values in order to draw a histogram and bubble chart. 


\section{Results}

\subsection{Acquisition of Active Components and Potential Target Genes of Cortex phellodendri}

From the TCMSP database we obtained 10 active components of Cortex phellodendri, such as quercetin and rutaecarpine and so on, using the screening conditions of OB $>30 \%$ and DL $>0.18$ (Table 1). At the same time, we converted target genes corresponding to active components of Cortex phellodendri in the UniProt database.

Table 1. Active components of Cortex phellodendri.

\begin{tabular}{llllll}
\hline No. & molID & molName & MW & OB (\%) & DL \\
\hline 01 & MOL001454 & berberine & 336.39 & 36.86 & 0.78 \\
02 & MOL001458 & coptisine & 320.34 & 30.67 & 0.86 \\
03 & MOL002662 & rutaecarpine & 287.34 & 40.3 & 0.6 \\
04 & MOL002668 & Worenine & 334.37 & 45.83 & 0.87 \\
05 & MOL000358 & beta-sitosterol & 414.79 & 36.91 & 0.75 \\
06 & MOL000785 & palmatine & 352.44 & 64.6 & 0.65 \\
07 & MOL000787 & Fumarine & 353.4 & 59.26 & 0.83 \\
08 & MOL000098 & quercetin & 302.25 & 46.43 & 0.28 \\
09 & MOL002894 & berberrubine & 322.36 & 35.74 & 0.73 \\
10 & MOL006422 & thalifendine & 322.36 & 44.41 & 0.73 \\
\hline
\end{tabular}

There are 10 active components of Cortex phellodendri. molID=molecular identification number; molName $=$ molecular name; $\mathrm{MW}=$ molecular weight; $\mathrm{OB}=$ oral bioavailability; $\mathrm{DL}=$ drug-likeness.

\subsection{Predicting Potential Target Genes of Cortex phellodendri in the Treatment of Hemorrhoids}

We retrieved a total of 144 hemorrhoids-related genes from the GeneCards database and compared them with target genes corresponding to each active component of Cortex phellodendri. We selected 29 common target genes (Table 2) as potential target genes of Cortex phellodendri in the treatment of hemorrhoids (Figure 1).

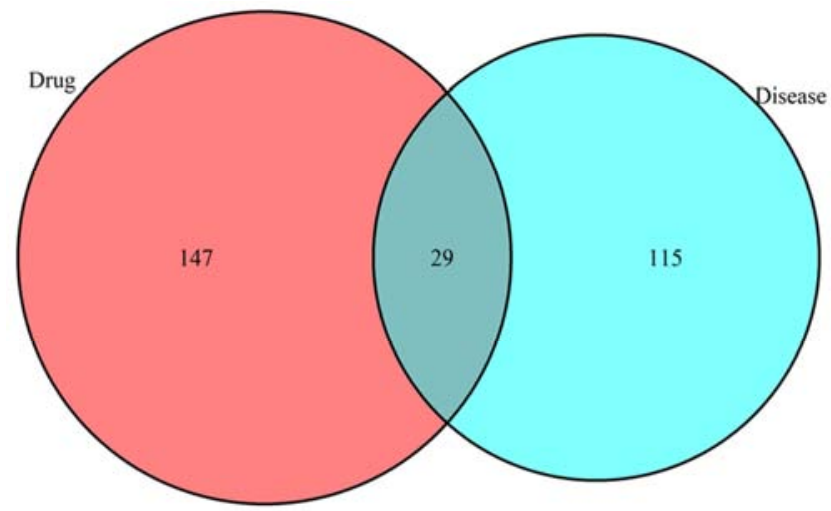

Figure 1. The target genes in common between Cortex phellodendri and hemorrhoids.

Table 2. Potential target information of Cortex phellodendri.

\begin{tabular}{|c|c|c|c|c|c|c|c|c|}
\hline No. & Uniprotkb & Target & No. & Uniprotkb & Target & No. & Uniprotkb & Target \\
\hline 1 & P35228 & NOS2 & 11 & P15692 & VEGFA & 21 & P49908 & SELE \\
\hline 2 & Q8N119 & $M M P 2$ & 12 & P22301 & IL 10 & 22 & P10145 & CXCL8 \\
\hline 3 & P14780 & $M M P 9$ & 13 & P05231 & IL6 & 23 & O15392 & BIRC5 \\
\hline 4 & P10415 & BCL2 & 14 & C9JW72 & TP63 & 24 & P60568 & IL2 \\
\hline 5 & Q07812 & $B A X$ & 15 & P03956 & $M M P 1$ & 25 & P08514 & PLAT \\
\hline 6 & P55211 & CASP9 & 16 & Q16665 & $H I F 1 A$ & 26 & P07204 & $T H B D$ \\
\hline 7 & P27169 & PON1 & 17 & P01106 & $M Y C$ & 27 & P02461 & COL $3 A 1$ \\
\hline 9 & P08254 & $M M P 3$ & 19 & P05362 & ICAMI & 29 & P29965 & $C D 40 L G$ \\
\hline 10 & P00533 & $E G F R$ & 20 & P13500 & CCL2 & & & \\
\hline
\end{tabular}

There are 29 target genes corresponding to active components of Cortex phellodendri in the UniProt database.

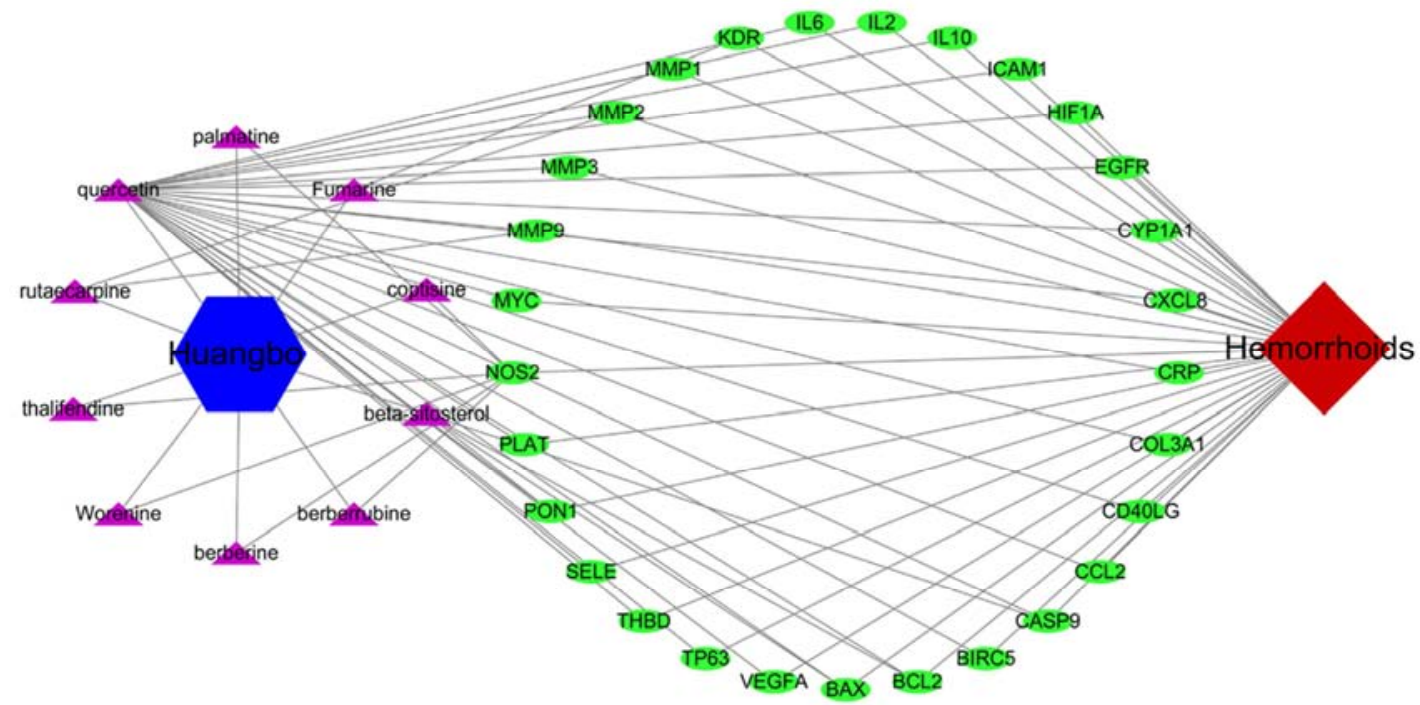

Figure 2. Construction of component-target gene-disease network. 


\subsection{Constructing a Component-target Gene-disease Network}

We introduced potential target genes of Cortex phellodendri for hemorrhoids treatment into Cytoscape to construct a component-target gene-disease network (Figure 2). The network had 29 nodes and 1190 strip edges. In Figure 2, the blue octagonal shape represents Cortex phellodendri, the purple triangle represents the active-component molecule, the green circle represents the target gene, and the red diamond represents the disease. As the figure shows, the same active ingredient can correspond to different target genes, and different active ingredients can correspond to the same target gene. This suggested that the potential mechanism of Cortex phellodendri in the treatment of hemorrhoids is mediated by multi-component and multi-target genes.

\subsection{Constructing a PPI Network and Screening Core Target Genes}

We imported potential target genes of Cortex phellodendri in hemorrhoids treatment into the STRING database and obtained a PPI network (Figure 3) and TSV file. With the help of $\mathrm{R}$ language, we calculated a genes. tsv file and obtained the Top 20 target genes of degree value (Figure 4).

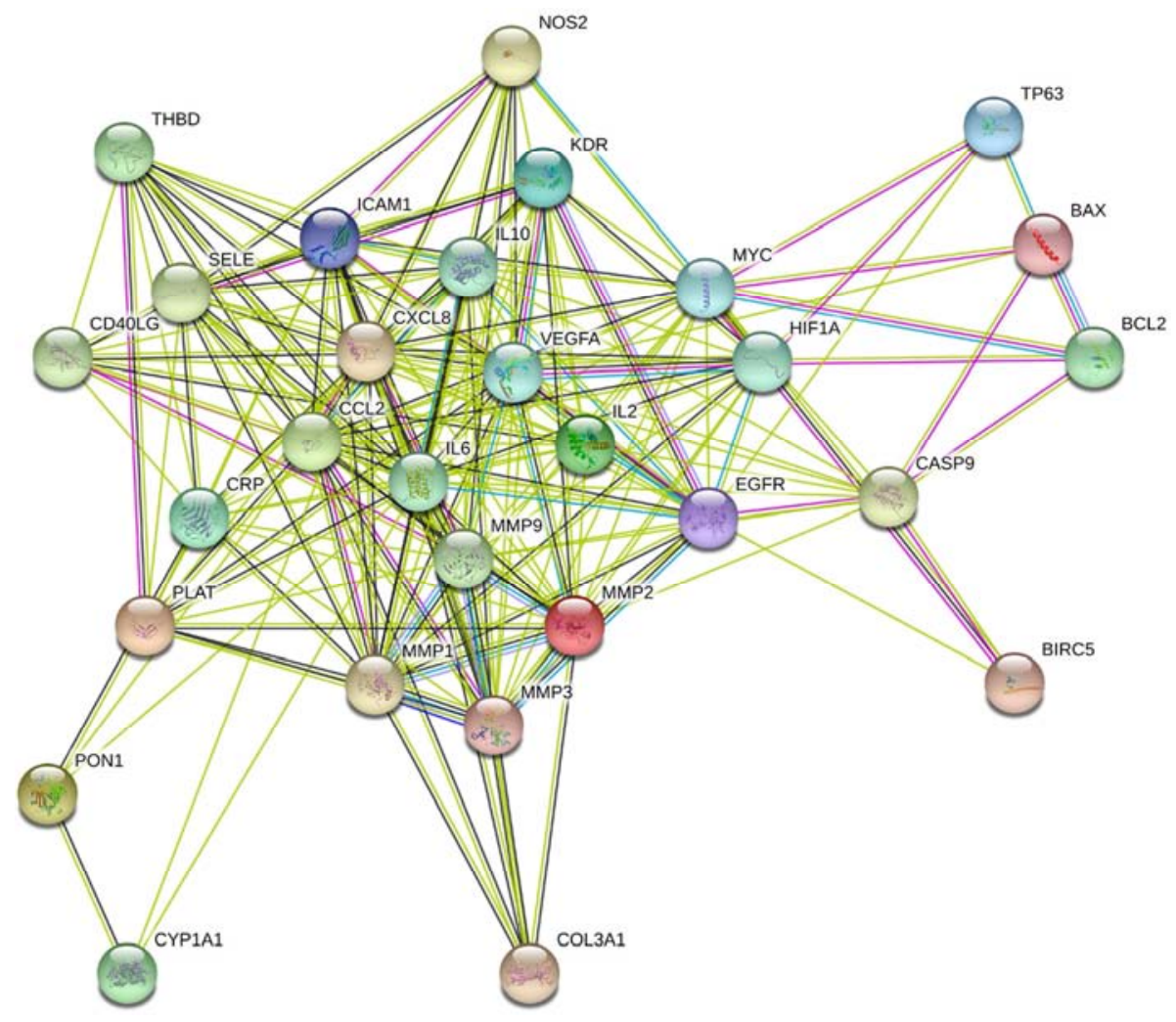

Figure 3. Construction of PPI network.

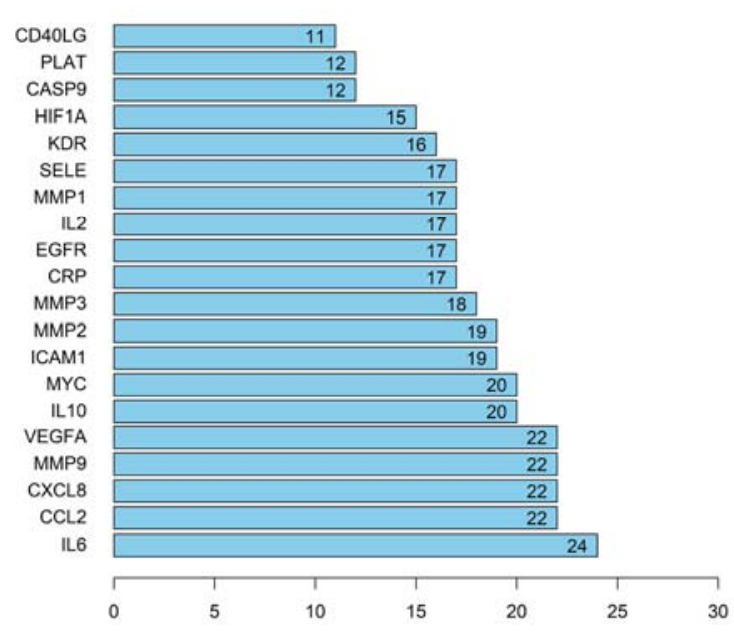

Figure 4. Top 20 core target genes.

\subsection{GO Function and KEGG Pathway Enrichment Analyses}

Using R language, we performed GO function and KEGG pathway enrichment analyses of potential target genes of Cortex phellodendri in hemorrhoids treatment. The results of GO function enrichment analysis (Figures 5 and 6) showed that the biological processes were mainly those such as serine-type endopeptidase activity, serine hydrodase activity, and so on. The cell components were mainly those such as cytokine receptor binding, heat shock protein binding, Hsp90 protein binding, and so on. The results of KEGG pathway enrichment analysis (Figures 7 and 8) showed that potential target genes of Cortex phellodendri in the treatment of hemorrhoids were mainly enriched in signaling pathways such as advanced glycation end products and receptor for advanced glycation end products (AGE-RAGE) signaling 
pathway in diabetic complications, hypoxia-inducible factor 1 (HIF-1), IL-17, and Relaxin.

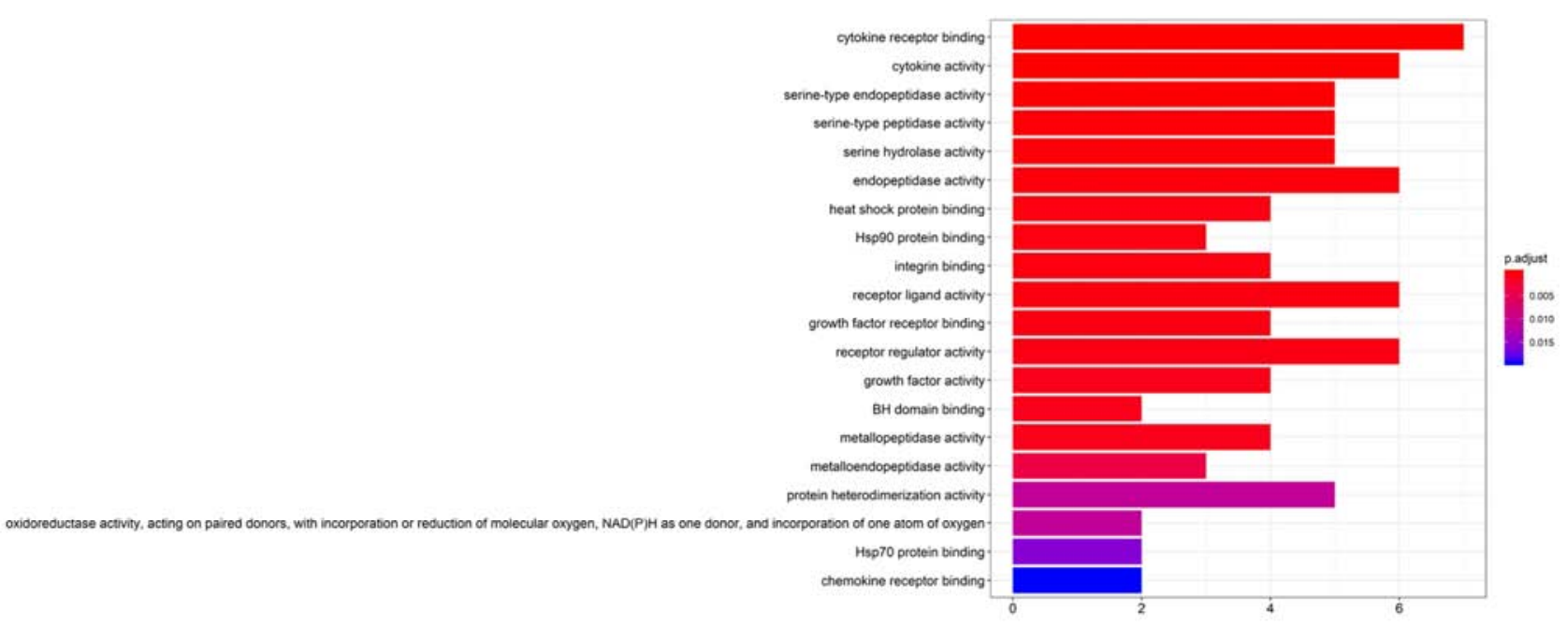

Figure 5. GO functional enrichment analysis (histogram).

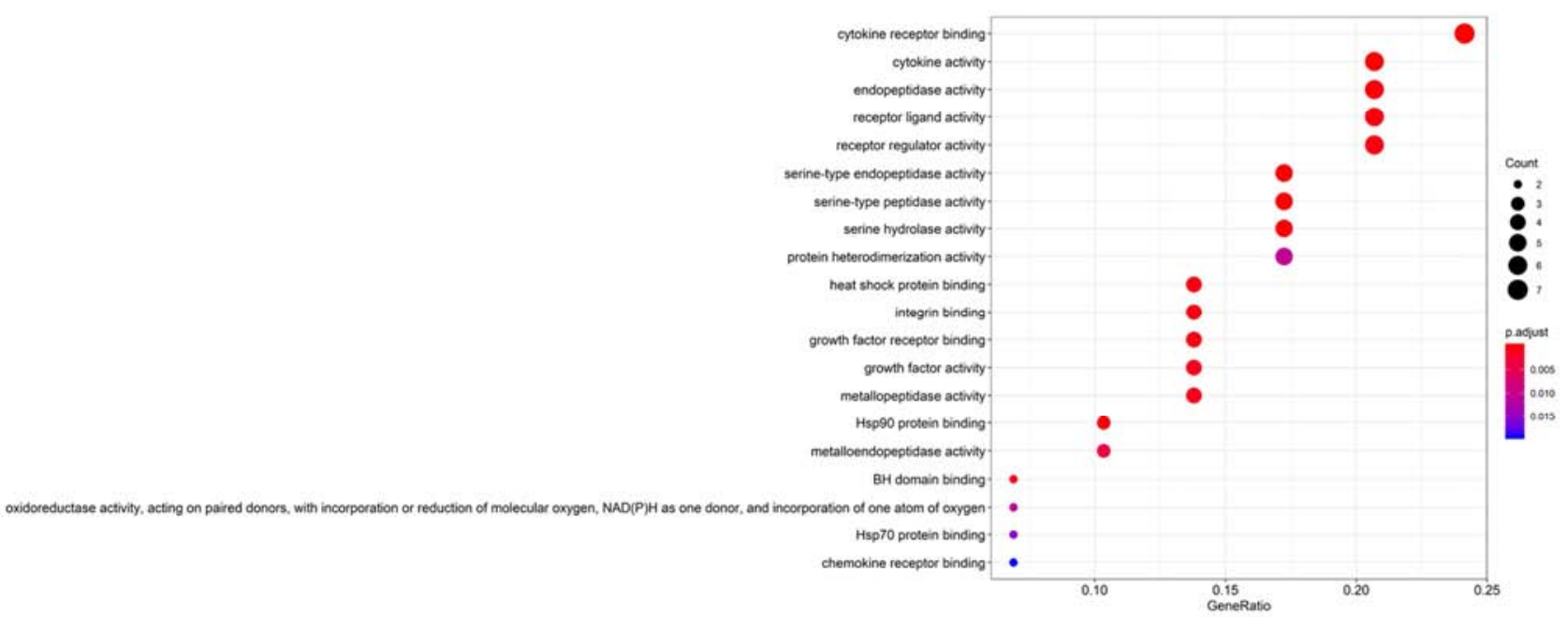

Figure 6. GO functional enrichment analysis (bubble chart).

\section{Discussion}

According to TCM, hemorrhoids are caused by eating disorders, dampness and heat blocking the intestines, and stasis over time [14]. Cortex phellodendri is a common medicine for clearing away heat and removing fire and blood stasis [15]. This indicates that Cortex phellodendri has the theoretical basis of TCM in the treatment of hemorrhoids. In fact, various preparations made by Cortex phellodendri have been widely used in clinical practice to treat anal diseases including hemorrhoids.

From Table 1 and Figure 2, we learn that the active ingredients of Cortex phellodendri are quercetin and rutaecarpine and berberine. Pharmacological studies have shown that quercetin can relieve smooth muscle spasm and improve skin edema [16]. rutaecarpine can improve vascular permeability and inhibit microthrombus formation [17]. berberine can accelerate wound healing by promoting angiogenesis and granulation growth of wound [18]; Quercetin can help to eliminate edema and improve local blood circulation so as to achieve the effect of reducing swelling [19]. Therefore, we hypothesized that the pharmacological action of Cortex phellodendri in the treatment of hemorrhoids may be achieved through the anti-inflammatory and hemostatic effect of quercetin, promoting angiogenesis, promoting the growth of granulation of wound of rutaecarpine, eliminating edema, improving local blood circulation of berberine, and so on.

It is not difficult to conclude from Figure 4 that IL-6, IL-8, CCL2, EGFR and IL-10 are the core genes of Cortex phellodendri in the treatment of hemorrhoids. IL-6, IL-8 and CCL2 are pro-inflammatory factors and IL-10 is antiinflammatory factor, according to literature studies [20-23]. IL-6 and CCL2 are important factors involved in immune response and inflammatory response [24]. Increased levels of IL-6, IL-8, CCL2, and IL-10 indicate injury and inflammation [25]. When hemorrhoids present symptoms, such as hemorrhoid prolapses, pain, bleeding, etc., IL-6, IL-8, 
CCL2, and IL-10 participate in the body's anti-inflammatory response and immune response under the feedback of the neurohumoral system, and their levels are correspondingly increased. The active ingredient of Cortex phellodendri may be to reduce inflammation, promote wound healing and relieve pain by inhibiting IL-6, IL-8, CCL2 and enhancing IL-10 and other cytokines.

Recent studies have found that the interaction between the receptor for advanced glycation end products (RAGE) and its ligand advanced glycation end products (AGE) is associated with vascular diseases [26, 27]. Hemorrhoids is believed to be caused by tortuous, dilated, hyperemia and other pathological changes in the anorectal vein. Therefore, RAGE-AGE may be related to the pathological changes of hemorrhoids. Increased AGE concentration in the human body may cause pathological changes in the anorectal vein $[28,29]$, thereby inducing symptoms of hemorrhoids. figures 7 and 8 show that the KEGG pathway of Cortex phellodendri in the treatment of hemorrhoids mainly involves RAGE-AGE, etc. Cortex phellodendri may inhibit RAGE-AGE to reverse hemorrhoid vascular lesions, which may lead to remission or reduction of hemorrhoids symptoms.

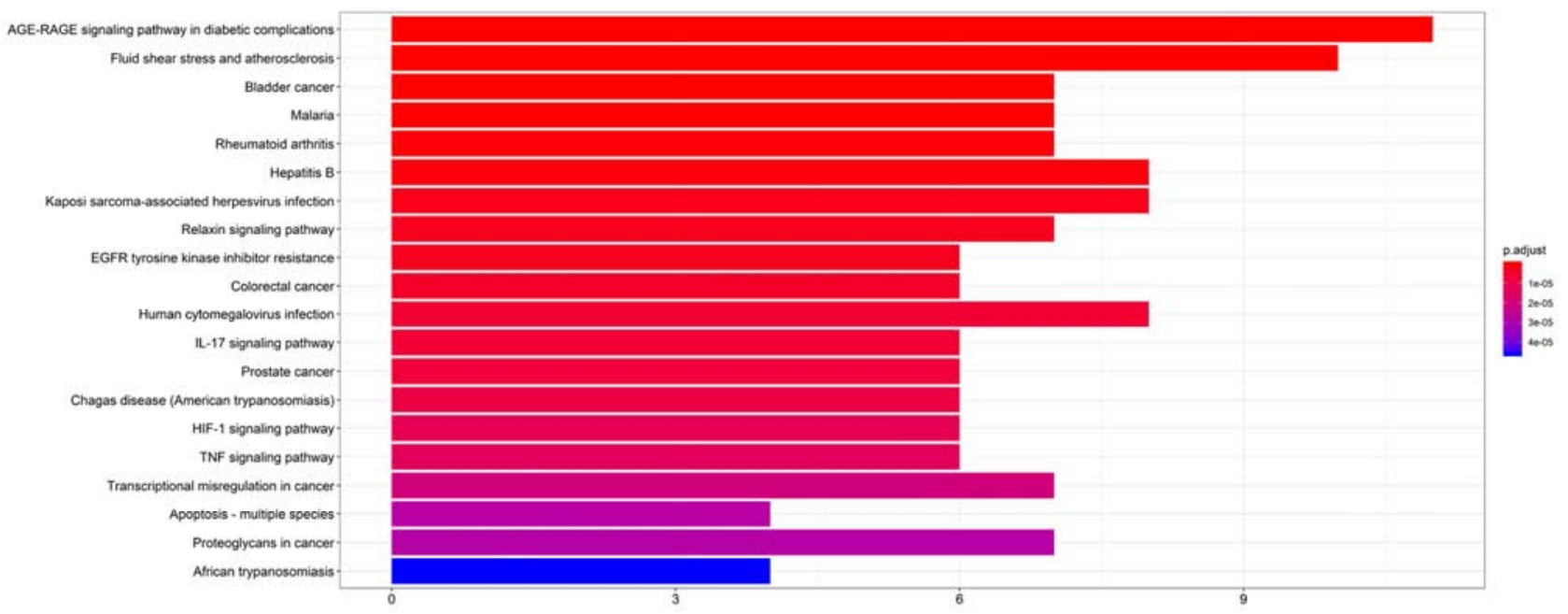

Figure 7. Results of KEGG pathway enrichment analysis (histogram).

To sum up, the effective components of Cortex phellodendri were quercetin and rutaecarpine and berberine. These active components may play a pharmacological role through the core gene IL-6, IL-8, CCL2, EGFR and IL-10 and the RAGE-AGE signaling pathway, thus achieving the therapeutic effect of hemorrhoids. The purpose of this study is to lay the foundation for the further study of the mechanism of action, and provide a new idea for the subsequent clinical improvement of acute hemorrhoid attack symptoms such as prolapse, pain, bleeding, etc.

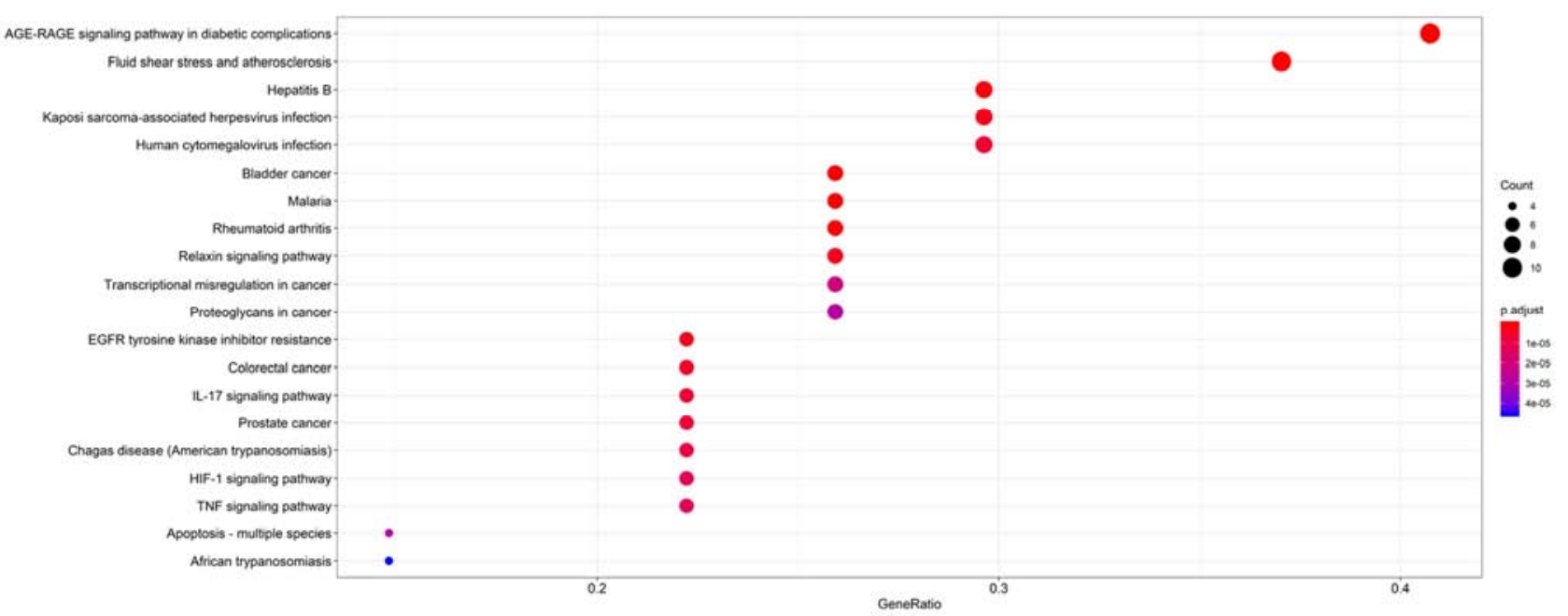

Figure 8. Results of KEGG pathway enrichment analysis (bubble chart).

\section{Conclusion}

Based on network pharmacology, Cortex phellodendri is expected to be mined as a candidate Traditional Chinese Medicine (TCM) for the treatment of hemorrhoids. Its mechanism for treating this disease operates via multiple components and pathways. This study provides the basic 
theory and the basis for further research.

\section{Data Availability}

The original data used to support this study can be available from the corresponding author upon request.

\section{Conflict of Interest}

The authors declare that they have no competing interests.

\section{Acknowledgements}

We thank LetPub for its linguistic assistance during the preparation of this manuscript.

\section{Reference}

[1] Muguruma N, Takayama T. Endoscopic sclerotherapy with aluminum potassium sulfate and tannic acid: An effective and less invasive strategy for internal hemorrhoids [J]. Clin Endosc, 2019, 52 (6): 521-522.

[2] Mishra S, Sahoo A K, Elamurugan T P, et al. Polidocanol versus phenol in oil injection sclerotherapy in treatment of internal hemorrhoids: A randomized controlled trial [J]. Turk J Gastroenterol, 2020, 31 (5): 378-383.

[3] Lee K Y, Lee J I, Park Y Y, et al. Hemorrhoids are associated with urinary incontinence [J]. J Womens Health (Larchmt), 2020, 29 (11): 1464-1468.

[4] Lambert L, Jahoda J, Grusova G, et al. Ct colonography has low sensitivity but high specificity in the detection of internal hemorrhoids [J]. Diagn Interv Radiol, 2020, 26 (2): 82-86.

[5] Shen K, Wang C, Gao Z D, et al. [procedure for prolapse and hemorrhoids versus stapled transanal rectal resection in the treatment of grade iv hemorrhoids] [J]. Zhonghua Wei Chang Wai Ke Za Zhi, 2019, 22 (12): 1165-1169.

[6] Ji L, Li L, Weng L, et al. Tissue selecting technique megawindow stapler combined with anal canal epithelial preservation operation for the treatment of severe prolapsed hemorrhoids: A study protocol for a randomized controlled trial [J]. Medicine (Baltimore), 2020, 99 (45): e23122.

[7] Xiongdong Zhong. An Exploration of the Active Ingredients of Salvia miltiorrhiza in the Treatment of Gastric Cancer and Its Mechanism Based on Network Pharmacology. Journal of Cancer Treatment and Research. Vol. 8, No. 2, 2020, pp. 34-44.

[8] Shi S Y, Zhou Q, He Z Q, et al. Traditional chinese medicine (liang-xue-di-huang decoction) for hemorrhoid hemorrhage: Study protocol clinical trial (spirit compliant) [J]. Medicine (Baltimore), 2020, 99 (16): e19720.

[9] Gan T, Liu Y D, Wang Y, et al. Traditional chinese medicine herbs for stopping bleeding from haemorrhoids [J]. Cochrane Database Syst Rev, 2010, (10): CD006791.

[10] Boost M, Yau P, Yap M, et al. Determination of cytotoxicity of traditional chinese medicine herbs, rhizoma coptidis, radix scutellariae, and cortex phellodendri, by three methods [J]. Cont Lens Anterior Eye. 2016, 39 (2): 128-132.
[11] Zhang Z, Zhang Y, Zhang Z, et al. Comparative analysis of DNA barcoding and hplc fingerprint to trace species of phellodendri cortex, an important traditional chinese medicine from multiple sources [J]. Biol Pharm Bull. 2016, 39 (8): 1325-1330.

[12] $\mathrm{Li} \mathrm{CY}, \mathrm{Lu} \mathrm{HJ}$, Lin $\mathrm{CH}$, et al. A rapid and simple determination of protoberberine alkaloids in cortex phellodendri by $1 \mathrm{~h} \mathrm{nmr}$ and its application for quality control of commercial traditional chinese medicine prescriptions [J]. J Pharm Biomed Anal. 2006, 40 (1): 173-178.

[13] Zhang DH, Zhang X, Peng B, et al. Network pharmacology suggests biochemical rationale for treating covid-19 symptoms with a traditional chinese medicine $[\mathrm{J}]$. Commun Biol. 2020, 3 (1): 466.

[14] Ren CP. Combined traditional chinese and western medicine sclerosing therapy in internal hemorrhoids $[\mathrm{J}]$. Chin Med J (Engl). 1977, 3 (2): 137-142.

[15] Zhou HY, Wang D, Cui Z. Ferulates, amurenlactone a and amurenamide a from traditional chinese medicine cortex phellodendri amurensis [J]. J Asian Nat Prod Res. 2008, 10 (56): 409-413.

[16] Kim SG, Sung JY, Kim JR, et al. Quercetin-induced apoptosis ameliorates vascular smooth muscle cell senescence through amp-activated protein kinase signaling pathway [J]. Korean J Physiol Pharmacol. 2020, 24 (1): 69-79.

[17] Surbala L, Singh CB, Devi RV, et al. Rutaecarpine exhibits anti-diabetic potential in high fat diet-multiple low dose streptozotocin induced type 2 diabetic mice and in vitro by modulating hepatic glucose homeostasis [J]. J Pharmacol Sci. 2020, 143 (4): 307-314.

[18] Chen L, Zhu L, Chen J, et al. Crystal structure-guided design of berberine-based novel chitinase inhibitors [J]. J Enzyme Inhib Med Chem. 2020, 35 (1): 1937-1943.

[19] Townsend EA, Emala CW, Sr. Quercetin acutely relaxes airway smooth muscle and potentiates beta-agonist-induced relaxation via dual phosphodiesterase inhibition of plcbeta and pde 4 [J]. Am J Physiol Lung Cell Mol Physiol. 2013, 305 (5): L396-403.

[20] Triantos C, Kalafateli M, Aggeletopoulou I, et al. Vitamin drelated immunomodulation in patients with liver cirrhosis $[\mathrm{J}]$. Eur J Gastroenterol Hepatol. 2020, 32 (7): 867-876.

[21] Milajerdi A, Mousavi SM, Sadeghi A, et al. The effect of probiotics on inflammatory biomarkers: A meta-analysis of randomized clinical trials [J]. Eur J Nutr. 2020, 59 (2): 633-649.

[22] Ohchi Y, Goto K, Yasuda N, et al. High efficiency removal of cytokines and hmgb-1 by continuous hemofiltration with a dual layered polyethersulfone membrane: An ex vivo study [J]. Ther Apher Dial. 2019, 23 (2): 173-179.

[23] Atilgan R, Pala S, Yavuzkir S, et al. What is the impact of short- and long-term supplementation of either cabergoline or clarithromycin on resolving rat ovarian hyperstimulation syndrome (ohss) model [J]. J Obstet Gynaecol. 2019, 39 (5): 687-694.

[24] Semeniuk LM, Likhachov VK, Yuzvenko TY, et al. Risk markers of reproductive loss in women with hyperandrogenism [J]. Wiad Lek. 2018, 71 (8): 1550-1553. 
[25] Catano Canizales YG, Uresti Rivera EE, Garcia Jacobo RE, et al. Increased levels of aim 2 and circulating mitochondrial DNA in type 2 diabetes [J]. Iran J Immunol. 2018, 15 (2): 142155 .

[26] Velayoudom-Cephise FL, Cano-Sanchez M, Bercion S, et al. Receptor for advanced glycation end products modulates oxidative stress and mitochondrial function in the soleus muscle of mice fed a high-fat diet [J]. Appl Physiol Nutr Metab. 2020, 45 (10): 1107-1117.

[27] Prasad K. Age-rage stress in the pathophysiology of pulmonary hypertension and its treatment [J]. Int $\mathrm{J}$ Angiol. 2019, 28 (2): 71-79.
[28] Isoyama N, Machowska A, Qureshi AR, et al. Elevated circulating s100a 12 associates with vascular disease and worse clinical outcome in peritoneal dialysis patients [J]. Perit Dial Int. 2016, 36 (3): 269-276.

[29] Kim JK, Park S, Lee MJ, et al. Plasma levels of soluble receptor for advanced glycation end products (srage) and proinflammatory ligand for rage (en-rage) are associated with carotid atherosclerosis in patients with peritoneal dialysis $[\mathrm{J}]$. Atherosclerosis. 2012, 220 (1): 208-214. 